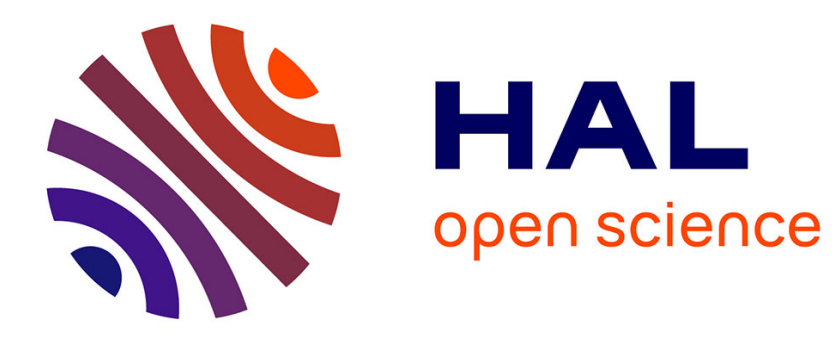

\title{
Framing Effects as Violations of Extensionality
}

Sacha Bourgeois-Gironde, Raphaël Giraud

\section{To cite this version:}

Sacha Bourgeois-Gironde, Raphaël Giraud. Framing Effects as Violations of Extensionality. Theory and Decision, 2009, 67 (4), pp.385-404. ijn_00432662

\section{HAL Id: ijn_00432662 \\ https://hal.science/ijn_00432662}

Submitted on 16 Nov 2009

HAL is a multi-disciplinary open access archive for the deposit and dissemination of scientific research documents, whether they are published or not. The documents may come from teaching and research institutions in France or abroad, or from public or private research centers.
L'archive ouverte pluridisciplinaire HAL, est destinée au dépôt et à la diffusion de documents scientifiques de niveau recherche, publiés ou non, émanant des établissements d'enseignement et de recherche français ou étrangers, des laboratoires publics ou privés. 


\title{
Framing effects as violations of extensionality
}

\author{
Sacha Bourgeois-Gironde · Raphaël Giraud
}

(C) Springer Science+Business Media, LLC. 2009

\begin{abstract}
Framing effects occur when different descriptions of the same decision problem give rise to divergent decisions. They can be seen as a violation of the decisiontheoretic version of the principle of extensionality (PE). The PE in logic means that two logically equivalent sentences can be substituted salva veritate. We explore what this notion of extensionality becomes in decision contexts. Violations of extensionality may have rational grounds. Based on some ideas proposed by the psychologist Craig McKenzie and colleagues, we contend that framing effects are justified when the selection of one particular frame conveys choice relevant information. We first discuss this idea from a philosophical point of view, and proceed next to formalize it first in the context of the Bolker-Jeffrey decision theory. Finally, we extend the previous analysis to non-expected utility theories using the Biseparable Preference model introduced by Ghirardato and Marinacci (2001) and therefore show that the analysis is independent of the assumptions of Bayesian decision theory.
\end{abstract}

Keywords Framing effects · Extensionality · Information processing · Bolker-Jeffrey decision model · Biseparable Preferences

\section{Introduction}

Framing effects occur when different descriptions of the same decision problem give rise to divergent decisions. They are a well-documented experimental fact, as shown

\footnotetext{
S. Bourgeois-Gironde

Institut Jean Nicod, Ecole Normale Supérieure, 29, rue d'Ulm, 75005 Paris, France

e-mail: Sacha.Bourgeois-Gironde@enslsh.fr

R. Giraud ( $\square)$

CRESE, University of Franche-Comté, 45 D avenue de l'Observatoire, 25000 Besançon, France e-mail: raphael.giraud@univ-fcomte.fr
} 
by surveys by Kühberger (1998) and Levin et al. (1998). They cover a great diversity of phenomena, related to the diversity of experimental conditions. ${ }^{1}$ However, a widely accepted, though seldom explicitly formulated, ${ }^{2}$ principle of decision-theoretic models states that preferences should not be affected by a variation in the description of the problem (Arrow 1982; Tversky and Kahneman 2000, Chap. 12). This principle is very often referred to as the invariance principle. Arrow calls it extensionality, which is an explicit reference to a principle of logic. In logic, the principle of extensionality requires of two formulas which have the same truth-value under any truth-assignments to be mutually substitutable salva veritate in a sentence that contains one of these formulas. It can thus be understood as the application of the invariance principle to logical contexts wherein truth-preservation is what is essentially at stake, but one can also envision invariance at a higher level of generality, encompassing decision-theoretic contexts. In the latter case, it is not only truth, but more generally information relevant to the coherent application of principles of choice among various alternatives, which is considered. The invariance principle then sets up a constraint on what must count as equivalent pieces or states of information from the point of view of the exercice of rational choice. Sen (1986, Chap. 2) spells out this notion of invariance as exerting an informational constraint on what objects of choice count as equivalent by introducing the idea of an isoinformation set containing objects of choice taken to be similar in terms of relevant information and which will be consequently treated in the same way in actual choices and judgements. Of course, one can easily remark that the notion of what is informationally relevant is crucial here. It will determine what objects belong to the same isoinformation set. It is precisely inherent to an invariance principle to specify under what dimension or characterization objects will be taken to be isoinformative. Once this notion of informational relevance and invariance is fixed in the particular case of decision contexts, violations of extensionality in those contexts mean that irrelevant information appears to determine the choices or judgments that are being made. Framing effects can then be defined as cases in which objects are being discriminated and treated in a way that is not in accordance with the informational constraint embodied by the invariance principle normally usually assumed to prevail in the contexts at hand.

Different interpretations have been proposed for framing effects under their various guises, mainly from a purely psychological point of view. Because they have long been discarded as irrational and because formal models of decision theory have for a long time only cared about rational behavior, there are very few formal models of framing effects. Obviously the introduction of framing effects into formal models entails a recasting of classical decision-theoretic models. In the most famous of these formal models, Prospect Theory (Kahneman and Tversky 1979,

\footnotetext{
1 Levin et al. (1998) thus distinguish three kinds of framing effects, based on what is manipulated:

- risky choice framing: manipulation of the consequences of risky prospects,

- attribute framing: manipulation of one attribute of the objects to be evaluated,

- goal framing: manipulation of the goal of a given action or behavior.

2 See Cohen and Jaffray (1980), Lipman (1999), Rubinstein (2000), and Le Menestrel and Van Wassenhove (2001) for some explicit formulations.
} 
Tversky and Kahneman 1992), framing effects motivate the introduction of reference dependence and of different behaviors in the domains of gains and losses-specifically, risk aversion in the domain of gains and risk propensity in the domain of losses. In this account of framing effects, different frames lead to the selection of different reference points, hence to a different encoding of outcomes as gains or losses and therefore to different choices.

Until recently, this model remained the only one for framing effects. Some alternative models currently emerge. Gold and List (2004) base their account of framing effects on a decision-making process in which decisions are the result of the acceptance or rejection of a set of background propositions, which constitute the reasons for decision. Giraud (2004) develops an axiomatic model of framing effects and shows that they occur because the decision maker perceives some missing information in the decision problem and different frames lead him to attribute different probabilities to the implicit states of the world corresponding to the missing information. A similar idea can be found in Ahn and Ergin (2007) where the contingencies can be framed in different ways in the sense that some events may or may not be distinguished, i.e., foreseen by the decision maker, and not foreseeing some events makes a difference.

In this article, we pursue this line of explanation and rationalization of framing effects in the context of two well-established decision models: the Bolker-Jeffrey (proposition-based) decision model, which is a Bayesian model, and the Biseparable Preference decision model of Ghirardato and Marinacci (2001), which is a non-Bayesian and very general model accommodating the Ellsberg paradox. By investigating the source of extensionality in the Bolker-Jeffrey model, we will see why it cannot simply accommodate framing effects. As we will show, Bolker-Jeffrey decision theory implicitly relies on the entailment of indifference from logical equivalence. Our bypassing strategy will then be to formalize in the original Bolker-Jeffrey framework the idea that two propositions refer to the same state of the world, a notion we shall call coreportiveness. We will then show how this allows, first, that coreportive propositions may not belong to the same isoinformation set (in the sense of Sen 1986) and how this implies violations of extensionality. We will then proceed to extend the approach followed in the context of the Bolker-Jeffrey model to the Ghirardato-Marinacci model, in order to show that this approach is perfectly compatible with accommodating the Ellsberg paradox.

\section{Extensionality in the Bolker-Jeffrey model}

In this section, we shall first review the basic features of the Bolker and Jeffrey decision model and then single out what is arguably the true source of extensionality in the model.

\subsection{The Bolker-Jeffrey decision model}

The most distinctive feature of Bolker and Jeffrey's decision model is that in this model, preferences are interpreted as bearing on propositions. As a matter of fact, 
they bear on non-null elements of a complete and atomfree Boolean algebra, ${ }^{3}$ that are interpreted as propositions. Recall the following definitions: a Boolean algebra is a tuple $\langle\mathscr{A}, \wedge, \vee, \neg, \top, \perp\rangle$ where $\mathscr{A}$ is a nonempty set, $\wedge, \vee$ two commutative, associative, and distributive with respect to each other binary operations, $\neg$ a unary operation, and $\top$ and $\perp$ two elements of $\mathscr{A}$ satisfying ${ }^{4}$

(i) $a \vee(a \wedge b)=a$ and $a \wedge(a \vee b)=a$;

(ii) $a \wedge \neg a=\perp$ and $a \vee \neg a=\top$.

The canonical example of a Boolean algebra is the set $2^{X}$ of all subsets of a given set $X$ together with the natural set operations of union, intersection, and complementation. A Boolean algebra is complete if the binary operations can be performed on an arbitrary family of elements (i.e., if $\left(a_{i}\right)_{i \in I}$ is a family of elements of $\mathscr{A}$, then $\bigvee_{i \in I} a_{i} \in \mathscr{A}$ and $\left.\bigwedge_{i \in I} a_{i} \in \mathscr{A}\right)$. A Boolean algebra is naturally endowed with a partial order defined by $a \leq b$ if and only if $a \vee b=b$. It is atomfree if for any $a \in \mathscr{A}$, there exists $a^{\prime}, a^{\prime \prime}$ such that $a^{\prime}, a^{\prime \prime} \leq a, a^{\prime}, a^{\prime \prime} \neq a$ and $a=a^{\prime} \vee a^{\prime \prime}$. Note that the interpretation of elements of a Boolean algebra as propositions requires some manipulation on which we shall come back later on, since extensionality heavily relies on this identification.

Let $\langle\mathscr{A}, \wedge, \vee, \neg, \top, \perp\rangle$ be the Boolean algebra of interest and $\mathscr{A}^{\prime}:=\mathscr{A} \backslash\{\perp\}$. We consider a binary relation $\succsim$ defined on $\mathscr{A}^{\prime}$ representing preferences. Let $a$ and $b$ be two elements of $\mathscr{A}$. The phrase " $a \succsim b$ " must be understood as expressing the fact that the decision maker prefers that the proposition $a$ to be true rather than the proposition $b$. Therefore, this statement of preference may be understood as saying that the decision maker prefers the world to be in a certain state described by $a$ to its being in the state described by $b$.

This preference relation is assumed to satisfy the following axioms.

BJ $1 \succsim$ is complete and transitive.

Two elements $a, b$ are disjoint if $a \wedge b=\perp$.

BJ 2 For all disjoint $a, b \in \mathscr{A}^{\prime}, \quad a \succ b \Rightarrow a \succ a \vee b \succ b$ and $a \sim b \Rightarrow a \sim$ $a \vee b \sim b$.

BJ 3 For all pairwise disjoint $a, b, c \in \mathscr{A}^{\prime}$, if $a \sim b \nsucc c$ and $a \vee c \sim b \vee c$ then, for all $d$ disjoint from $a$ and $b, a \vee d \sim b \vee d$.

BJ 4 For any monotone sequence $\left(a_{n}\right)$ in $\mathscr{A}^{\prime}$, converging to $a$ (i.e., $a=\bigvee a_{n}$ or $\left.a=\bigwedge a_{n}\right)$, if $b \succ a \succ c$, then there exists $N \in \mathbb{N}$ such that $b \succ a_{n} \succ c$ for all $n \geq N$.

We now give the version of Bolker's theorem that will be of use in the sequel of this article.

Theorem 1 Under axioms BJ 1 to BJ 4, there exist a function $U: \mathscr{A}^{\prime} \rightarrow \mathbb{R}$ and a probability measure $P: \mathscr{A}^{\prime} \rightarrow[0,1]$ such that, for all $a, b \in \mathscr{A}^{\prime}$

\footnotetext{
${ }^{3}$ An example of a complete atomfree Boolean algebra is the set of subsets of $\mathbb{R}$ that are equal to the closure of their interior.

${ }^{4}$ See, e.g., Koppelberg (1989).
} 
1. $U(a) \geq U(b) \Longleftrightarrow a \succsim b$;

2. $U(a)=U(a \wedge b) P(b \mid a)+U(a \wedge \neg b) P(\neg b \mid a)$, with $P(b \mid a):=\frac{P(a \wedge b)}{P(a)}$.

As can be seen in condition 2, this theorem axiomatizes a version of expected utility, which shall be of further use in the sequel.

\subsection{Why are violations of extensionality precluded in the Bolker-Jeffrey model}

When reviewing the basic features of the Bolker-Jeffrey model, we alluded to the fact that the interpretation of elements of a Boolean algebra as propositions was not completely straightforward, and that it was related to extensionality. We shall now make this point clearer. Let $\mathbb{F}$ denote the set of formulas of a classical propositional language. The conjunction and disjunction operators, $\wedge$ and $\vee$, and the negation operator $\neg$, together with their classical properties, are natural candidates to make of $\mathbb{F}$ a Boolean algebra. However, in classical logic, all the properties of Boolean algebras are satisfied with the restriction that all equality signs in their statement must be replaced by the bi-conditional $\leftrightarrow$. Therefore, strictly speaking $\mathbb{F}$ is not a Boolean algebra. However, it is very close to be one. Let us see how the connection is made. Let $T$, the set of classical truth functions, be the set of functions $t: \mathbb{F} \rightarrow\{0,1\}$ satisfying $t(\alpha \wedge \beta)=\min \{t(\alpha), t(\beta)\}$ and $t(\neg \alpha)=1-t(\alpha)$. We say that $\alpha$ is logically equivalent to $\beta$, denoted $\alpha \equiv \beta$ whenever $t(\alpha)=t(\beta)$ for all $t \in T$. This defines an equivalence relation. Let $a=[\alpha]$ be the equivalence class of $\alpha$ (and $b$ the equivalence class of $\beta$ ). $a$ is the proposition expressed by sentence $\alpha$. Let $\mathbb{P}:=\mathbb{F} / \equiv$ be the set of propositions. Defining

$$
a \wedge b:=[\alpha \wedge \beta], \quad a \vee b:=[\alpha \vee \beta], \quad \perp:=[\perp], \quad \top:=[\top] \quad \text { and } \quad \neg a:=[\neg \alpha]
$$

makes $\mathbb{P}$ into a Boolean algebra, the Boolean algebra of classical logic. Assuming the appropriate additional properties for $\mathbb{F}$ and $T$, one would easily ensure that this Boolean algebra be complete and atomfree.

This construction shows that, if one identifies a formula and an element of a Boolean algebra, one is in fact implicitly neglecting any morphological differences between logically equivalent sentences or formulas, as it identifies a sentence with its equivalence class, the proposition that it expresses. This is what is done in the Bolker-Jeffrey decision model and this automatically yields extensionality in the strong sense defined above.

Another way of understanding this is to view extensionality as an implicit axiom of the theory: let us consider a preference relation $\succsim_{\mathbb{F}}$ on $\mathbb{F}$. In order to be able to apply Bolker's theorem to derive utilities and probabilities on sentences, one must first transfer the preference relation from $\mathbb{F}$ to $\mathbb{P}$ to construct them on propositions. A natural way of doing this is to define

$$
[\alpha] \succsim_{\mathbb{P}}[\beta] \Longleftrightarrow \alpha \succsim_{\mathbb{F}} \beta
$$


but this relation is ill-defined on $\mathbb{P}$ unless one assumes the following consistency requirement: $:^{5}$

$$
\forall a, b \in \mathbb{F}, \quad a \equiv b \Rightarrow a \sim_{\mathbb{F}} b
$$

This axiom is exactly the extensionality principle and, as coreportive sentences are logically equivalent, it precludes framing effects. Therefore, violations of extensionality are precluded in the Bolker-Jeffrey logic of decision. Is there, however, a way of bypassing this result?

\section{Bypassing extensionality in the Bolker-Jeffrey decision model}

\subsection{The main idea}

As we have just seen, extensionality in the Bolker-Jeffrey decision model is forced onto us by an implicit axiom implied both by the mathematical structure of the set on which preferences are defined, and by the interpretation of this set as a set of propositions. In order to bypass extensionality, therefore, it is desirable to modify either this structure or its interpretation in an appropriate way. By appropriate, we mean essentially that this modification should meet the following requirements:

1. Preserve the interpretation of objects of choice as propositions;

2. Preserve the mathematical tractability (i.e., applicability of Bolker's theorem);

3. Allow for a formal definition of extensionality in the model.

The first two requirements amount essentially to the preservation of the Boolean structure, and we shall see in the formal development how this is easily done. Some words of comment on the third requirement are, however, in order. If one wants to be able to bypass extensionality, one must first make this axiom explicit. However, if one defines extensionality of preferences in the same spirit as logical extensionality, imposing indifference for logically equivalent sentences, this is impossible in the Bolker-Jeffrey setup, as logically equivalent sentences are collapsed into a single object: the proposition they express. It is possible, however, to use another property of the logically equivalent sentences involved in framing effects: the fact that they refer to the same event.

\subsection{The formal model}

We shall consider two sets, $\mathscr{E}$ and $\mathbb{P}$, that we assume, like in the Bolker-Jeffrey decision model, to be atomfree and complete Boolean algebras. Elements of $\mathscr{E}$ are the possible events of interest in a given decision problem. Elements of $\mathbb{P}$ are to be interpreted as

\footnotetext{
5 The situations here bear some resemblance to the unearthing of the implicit independence condition in von Neumann and Morgenstern's axiomatization of Expected Utility. They worked directly on utilities, and defined mixtures of utilities. For this definition to be well defined, an implicit consistency axiom was required (see Malinvaud (1952)).
} 
propositions describing a given event, which in turn is an element of $\mathscr{E}$. To make this connection formal, we shall define a frame as a Boolean homomorphism from $\mathscr{E}$ to $\mathbb{P}$. Recall that a homomorphism between two Boolean algebras $\mathscr{A}$ and $\mathscr{B}$, is a mapping $\varphi: \mathscr{A} \rightarrow \mathscr{B}$ such that:

$$
\varphi\left(a \wedge a^{\prime}\right)=\varphi(a) \wedge \varphi\left(a^{\prime}\right) \text { and } \varphi(\neg a)=\neg \varphi(a) .
$$

It follows from this definition that

$$
\varphi\left(a \vee a^{\prime}\right)=\varphi(a) \vee \varphi\left(a^{\prime}\right), \quad \varphi(\perp)=\perp \quad \text { and } \varphi(\top)=\top .
$$

We define a decision problem with framing as a tuple $\langle\mathscr{E}, \mathbb{P}, \Phi, \succsim\rangle$, where $\Phi$, the set of frames, is a nonempty set of homomorphisms from $\mathscr{E}$ to $\mathbb{P}$ and $\succsim$ is a binary relation defined over the set $\mathbb{P}^{\prime}=\mathbb{P} \backslash\{\perp\}$ of noncontradictory propositions.

Let us now illustrate how the formalism fits the intuition of framing effects, and more precisely how a frame and a proposition are conceptually different. Consider first, the familiar example of the half-empty/hall-full glass. The event corresponding to this glass could be identified with the quantity of water the glass contains and the quantity it can contain. This is $e$. Now this event can be described according to two different frames: a positive frame, $\varphi^{+}$, according to which it is half-full, and a negative frame, $\varphi^{-}$, according to which it is half-empty. Consider now the proposition "The glass is half-full" $\left(s^{+}\right)$. This proposition can be interpreted as being $\varphi^{+}(e)$, i.e., the event $e$ described in the positive frame. Conversely, the proposition $s^{-}:$" the glass is half-empty" can be viewed as $\varphi^{-}(e)$. We can now consider the same glass with half as much water, event $e^{\prime}$. Now $\varphi^{+}\left(e^{\prime}\right)$ is "the glass is one quarter-full" and $\varphi^{-}\left(e^{\prime}\right)$ is "the glass is three quarters-empty".

Let us take another example that is more clearly related to a decision problem. This is an example by Quattrone and Tversky (2000, Chap. 25). Consider economic policies characterized by employment, unemployment, and inflation rates. Policy A yields $90 \%$ of employment and $12 \%$ of inflation. Policy B yields $95 \%$ of employment and $17 \%$ of inflation. Policy C yields $10 \%$ of unemployment and $12 \%$ of inflation. Policy D yields $5 \%$ of unemployment and $17 \%$ of inflation. The problem is to choose the best policy. Clearly, policies A and C can be seen as describing the same event. However, they describe it using different frames, the employment frame and the unemployment frame. The same can be said of B and D.

The principle of extensionality can be expressed in this framework:

Extensionality Principle The preference relation $\succsim$ satisfies the Extensionality Principle if, for all $e, e^{\prime} \in \mathscr{E}$, for all $\varphi, \varphi^{\prime}, \psi, \psi^{\prime} \in \Phi$, then

$$
\varphi(e) \succsim \varphi^{\prime}\left(e^{\prime}\right) \Longleftrightarrow \psi(e) \succsim \psi^{\prime}\left(e^{\prime}\right)
$$

For any $p \in \mathbb{P}$, if there exist a frame $\varphi$ and an event $e$ such that $p=\varphi(e)$, then $e$ will be said to be the reference of proposition $p$ relative to frame $\varphi$. We call coreportive two propositions with the same reference. The extensionality principle therefore 
implies that substituting coreportive propositions one for the other does not modify the preference ordering.

In order to state the result that shows that extensionality has been bypassed, we need to introduce the following concept:

Definition 1 Let $e$ be an event. An event $k$ is a good news for $e$ if:

(i) $\varphi(e \wedge k) \sim \varphi^{\prime}(e \wedge k)$, for all $\varphi, \varphi^{\prime} \in \Phi$;

(ii) $\varphi(e \wedge \neg k) \sim \varphi^{\prime}(e \wedge \neg k)$, for all $\varphi, \varphi^{\prime} \in \Phi$;

(iii) $\varphi(e \wedge k) \succ \varphi(e \wedge \neg k)$ for all $\varphi \in \Phi$.

Intuitively, a good news for a given decision is an information that we would rather have prior to making the decision and that helps us make the right decision. Now, from the formal definition of the concept of good news, it is clear that prior to deciding whether he/she should go for decision $e$ (i.e., decide that $e$ be the true event), the decision maker would like to know if $k$ obtains or does not obtain, because, if he/she knew it, he/she would know if he/she would be better off by choosing $e$ or by refraining from doing so: he/she is better off when choosing $e$ if and only if $k$ obtains, irrespective of the frame (property (iii)). Moreover, this is a piece of information that really concerns $e$ because in fact the utility that the decision maker will experience in the case that he/she has chosen $e$ and that $k$ obtains (respectively, does not obtain) shall be the same irrespective of the frame (properties (i) and (ii)). Therefore, knowing whether $k$ is true is really knowing something about real consequences of the decision. In some sense, $k$ forms the basis for an argument in favor of or against the choice of $e$ : if $k$ is true, then one should go for $e$; if $k$ is not true, then one should refrain from doing so.

The psychologists Sher and McKenzie (2006) have recently defended a view of framing effects according to which, even though subjects are aware of the coreportiveness of two propositions, $A$ and $B$, these propositions may not convey the same information for the subjects as soon as the speaker has chosen one formulation instead of the other, as these formulations may license different inferences. In Sher and McKenzie's terms: "Suppose (...) that speaker's conversational behavior exhibits the following regularity: speakers, choosing between uttering ' $A$ ' and uttering ' $B$ ', are more likely to utter ' $A$ ' when some background condition $C$ (not explicitly specified in the statements $\mathrm{A}$ and $\mathrm{B}$ ) holds than when $\mathrm{C}$ fails. In that case, a listener who hears a speaker say 'A' can safely infer a higher probability of $\mathrm{C}$ being true than if the speaker had said ' $\mathrm{B}$ ' (that is, $p(C \mid$ speaker says ' $\mathrm{A}$ ' $)>p(C \mid$ speaker says ' $\mathrm{B}$ '). If knowledge about the background condition $\mathrm{C}$ is relevant to the choice at hand, then the speaker's (e.g., experimenter's) utterance of the two logically equivalent statements A and B may with impunity lead to different decisions" (Sher and McKenzie 2006). Sher and McKenzie's idea is that different frames provide different clues to the relevant information. In other words, if two propositions are not indifferent, it is because the frames in which their common reference is described do not convey the same probabilistic information about the relevant pieces of information.

In particular, Sher and McKenzie (2006) have experimentally demonstrated the following conversational regularity: speakers usually choose the frame that emphasizes the dimension that has increased relative to some reference or average situation. For 
instance, when asked to describe an R\&D team in terms of its number of successes or of its number of failures, subjects tended to describe outstandingly good teams in terms of their number of successes while they tended to describe outstandingly bad teams in terms of their number of failures, even when the number of failures and successes was the same for each team (for instance, 20 failures and 30 successes). Now it is quite clear that, for somebody who has to make a funding decision for the R\&D team in question, knowing whether it is a good or a bad team is relevant. Let us call $k$ the event that the team is good. In our context, therefore, if the decision to be made is to fund (e) or not to fund $(\neg e)$, then the decision maker is better off if he/she chooses to fund while the team is good $(e \wedge k)$ than if he/she chooses to fund while the team is bad $(e \wedge \neg k)$, and this welfare ordering is independent of the way the team was described. For completeness, let us mention what would be the propositions at stake. There are two frames here: success $\left(\varphi^{+}\right)$and failure $\left(\varphi^{-}\right) . \varphi^{+}(e)$ corresponds to the proposition "funding a team that has had 30 successes", while $\varphi^{-}(\neg e)$ corresponds to "not funding a team that has had 20 failures". Given the conversational regularities empirically observed, both correspond to good decisions. Finally, $\varphi^{-}(e \wedge \neg k)$ corresponds to the proposition "funding a team that is not good and that has had 20 failures". $k$ is indeed an example of a good news.

As another example, consider again policies $\mathrm{C}$ and $\mathrm{D}$ in the macroeconomic policy example. Based on the conversational regularity recalled above, it could be inferred from the fact that the policy is described in terms of employment that the corresponding level of inflation is usually associated with a lower level of employment. On the contrary, framing this policy in terms of unemployment suggests this level of inflation usually leads to a lower rate of unemployment. ${ }^{6}$ Now, knowing which one of these statements is true is relevant for the decision at hand. Formally, let $k$ be the event "a $12 \%$ rate of inflation usually corresponds to a rate of employment less than $90 \%$ and a rate of unemployment greater than 10\%". Let $e$ be the event described by both $A$ and $C$. Clearly, $e \wedge k$ is preferred to $e \wedge \neg k$. Therefore, $k$ can be considered to be good news for $e$.

Let $\mathscr{K}(e)$ be the set of good news for $e$. The following theorem holds (proof in the Appendix).

Theorem 2 Let the structural assumption hold. Then, if $\succsim$ satisfies axioms BJ1 to $B J 4$, for each $\varphi \in \Phi$, there exists a (finitely additive) probability measure $P_{\varphi}$ on $\mathscr{E}$ such that, for all $\varphi, \varphi^{\prime} \in \Phi$, for all $e \in \mathscr{E}$, for all $k \in \mathscr{K}(e)$,

$$
\varphi(e) \succsim \varphi^{\prime}(e) \Leftrightarrow P_{\varphi}(k \mid e) \geq P_{\varphi^{\prime}}(k \mid e) .
$$

As it is easy to see, this theorem offers a way of bypassing extensionality. It gives indeed the conditions under which the decision maker is indifferent between two

\footnotetext{
6 One may wonder why it is not possible to infer that this level of unemployment usually corresponds to a lower level of inflation. Both interpretations would agree in the sense that they both indicate that the policy presented corresponds to a bad unemployment/inflation trade-off with respect to the usual situation. The idea is that there is no flexibility for framing the level of inflation in a positive or a negative frame in this example, so that only the choice of the unemployment frame is significant: unemployment has to be the dimension that has increased.
} 
coreportive propositions. These conditions, which need not hold, are exactly the ones suggested by Sher and McKenzie in their defense of the normative status of framing effects: they are indifferent if and only if the frames in which their common reference is expressed convey exactly the same information about choice-relevant pieces of information: here good news about their reference.

Let us come back to the macroeconomic example to illustrate. As we said, it can be inferred from the speaker's choice of A that $k$ is more likely than not $\neg k$. Similarly, it can be inferred from the speaker's choice of $\mathrm{C}$ that $\neg k$ is more likely than $k$. Let $e_{A C}$ be their common event and $\varphi^{e}$ and $\varphi^{u}$ be the employment and unemployment frames. Then $A=\varphi^{e}\left(e_{A C}\right)$ and $C=\varphi^{u}\left(e_{A C}\right)$. We therefore have that $P_{\varphi^{e}}\left(k \mid e_{A C}\right)>\frac{1}{2}$ and $P_{\varphi^{u}}\left(k \mid e_{A C}\right)<\frac{1}{2}$, therefore $P_{\varphi^{e}}\left(k \mid e_{A C}\right)>P_{\varphi^{u}}\left(k \mid e_{A C}\right)$, yielding $A \succ C$.

In the glass example, we can assume that the decision relevant information is whether the glass was originally empty. Here, the conversational regularity observed by Sher and McKenzie is that if a speaker describes the glass as half-full, that means that it was originally empty. Let $k$ be the event: "the glass was originally empty". This regularity can thus be written, with the notations of the example, $P_{\varphi^{+}}(k \mid e)>\frac{1}{2}$, and it also implies $P_{\varphi^{-}}(k \mid e)<\frac{1}{2}$ (the speaker would not say that the glass is half-full if it was previously full), and this implies that a half-full glass is preferred to a half-empty one.

It must be noticed, however, that, to the best of our knowledge, direct comparisons of the same event framed in different ways have not been tested, although, as it can be seen, predictions could be made on this comparison based on the insights captured by Theorem 2. This is especially interesting if one wants to compare it with Prospect Theory. Prospect Theory makes predictions for behavior within a given frame, the gain frame or the loss frame. It does not make any prediction across frames. In that sense, both Prospect Theory and the theory described above are partial theories of framing effects and they cannot be directly tested one against the other. So in principle, we would need a combination of both to account for framing under risk.

We would like, finally, to relate the model we have proposed to two other models of framing effects that we have mentioned in the introduction. According to Gold and List (2004), framing effects arise when two conditions are met: a logical one-that the order of consideration of the background propositions matters for the decision-, and an empirical one - that different frames lead to different orders of consideration. The authors show that the logical condition is equivalent to the existence of (implicit) inconsistencies in the decision maker's disposition to accept propositions, i.e., by the fact that there are contradictions in the set of background propositions the decision maker is ready to accept. The account we fleshed out can be summarized along similar lines: the empirical condition is that the weight of the arguments for or against a particular decision, as embodied by the probability of the truth of some favorable background condition, depends on the frame, and the logical condition, spelled out in the theorem, is that the outcome of the decision process depends on this probability. Following Tversky et al. (2000, Chap. 34) and Gold and List (2004) contrast value-based accounts of decision making, whereby decision is understood as the maximization of some function, and reason-based accounts, whereby it is the result of the weighing of arguments and inferences, and claim that value-based accounts are a subset of reason- 
based ones. Our formal model tends to show, in our opinion, that the difference is not so clear as it may seem, as formal, value-based models can be given an interpretation in terms of arguments. The account of framing effects in Giraud (2004) is based on the idea that agents perceived that there is some missing information in the decision problem, that the probability of this missing information depends on the frame, and that the outcome of the decision process depends on that probability. It is very close in spirit to the one we have given here, but the formalization is different, as it is based on a classical set up of decision under risk where objects of choice are lotteries. One major conceptual difference is that in this article the nature of the missing information is left implicit, whereas here we flesh it out in a rather precise way.

\section{Bypassing extensionality when ambiguity matters}

A substantial literature, both empirical and theoretical, now demonstrates and formally studies the impact of ambiguity in decision making under uncertainty, where by ambiguity it is understood the impossibility of assigning well-defined probabilities to at least one event, as opposed to the situation of risk (see Gilboa 2004 for references). The most appropriate model on which we can base an extension of the results above to a context where ambiguity matters is the biseparable preference model of Ghirardato and Marinacci (2001), because it is the weakest model allowing for a clear-cut separation between beliefs and preferences in the representation. Before describing this model, let us lay down the formal framework. Let $(S, \Sigma)$ be a measurable set of states of nature and $X$ be a set of outcomes. We assume that $X$ is a connected and separable topological space. Let $\mathscr{F}$ be the set of simple Savagean acts (or acts, for short), i.e., the set of functions $f: S \rightarrow X$ such that $f(S)$ is finite and for all $x \in X, f^{-1}(x) \in \Sigma$. Let $\succsim$ be a preference relation on $\mathscr{F}$. A bet is an act, $f$, such that there exist two outcomes $x \succ y$ and $A \in \Sigma$ such that $f(A)=\{x\}$ and $f\left(A^{c}\right)=\{y\}$. We denote $x A y$ for such an act.

Let $\succsim$ be a preference relation on $\mathscr{F}$. An event $A$ is said to be essential (resp., null, universal) for $\succsim$ if for some bet $x A y$, we have $x \succ x A y \succ y$ (resp., $x A y \sim y$, $x A y \sim x)$. The preference relation $\succsim$ is said to be biseparable if there exists a function $V: \mathscr{F} \rightarrow \mathbb{R}$, monotonic (i.e., $f(s) \succsim g(s)$ for all $s$ implies $V(f) \geqslant V(g)$ ) and unique up to increasing affine transformations whenever $\succsim$ has an essential event, and a capacity ${ }^{7} \rho_{V}: \Sigma \rightarrow[0,1]$ such that

$$
\begin{aligned}
f \succsim g & \Longleftrightarrow V(f) \geq V(g) ; \\
V(x A y) & =\rho_{V}(A) u_{V}(x)+\left(1-\rho_{V}(A)\right) u_{V}(y) \text { for all bets } x A y,
\end{aligned}
$$

where $u_{V}(x):=V(x)$. Ghirardato and Marinacci have shown that if $V^{\prime}$ is another such representation, then $u_{V}$ and $u_{V^{\prime}}$ are affinely related and $\rho_{V}=\rho_{V^{\prime}}$. We next recall the axiomatization of biseparable preferences by Ghirardato and Marinacci. They introduce the following axioms.

\footnotetext{
7 That is, a normalized $\left(\rho_{V}(S)=1\right.$ and $\left.\rho_{V}(\varnothing)=0\right)$ and monotonic $\left(\rho_{V}(A) \leq \rho_{V}(B)\right.$ whenever $\left.A \subseteq B\right)$ set function.
} 
GM $1 \succsim$ is complete and transitive, and there exist $f, g \in \mathscr{F}$ such that $f \succ g$ and an essential event $E$.

GM 2 (Dominance) For all $f, g \in \mathscr{F}, f(s) \succsim g(s)$ for all $s$ implies $f \succsim g$.

GM 3 (Eventwise monotonicity) For every nonnull $A$ and every $x, y \succsim z \in X$,

$$
x \succ y \Longrightarrow x A z \succ y A z .
$$

For every nonuniversal $A$ and every $x, y \precsim z \in X$,

$$
x \succ y \Longrightarrow z A x \succ z A y .
$$

GM 4 (Subcontinuity) Let $\left(f_{\alpha}\right)_{\alpha \in D}$ be a net and $f \in \mathscr{F}$ such that $f_{\alpha}$ and $f$ are all measurable with respect to the same finite partition, and $f_{\alpha}(s)$ converges to $f(s)$ for all $s \in S$. Then, for all $g \in \mathscr{F}, f_{\alpha} \succsim g$ (resp. $g \succsim f_{\alpha}$ ) implies $f \succsim g$ (resp. $g \succsim f$ ).

The last axiom uses a definition of subjective mixtures for acts that is as follows. The previous axiom and the connectedness of $X$ imply that any act $f$ admits a (unique up to indifference) certainty equivalent $c_{f}$. Let $f, g \in \mathscr{F}$ and $A \in \Sigma$. Define the statewise A-mixture of $f$ and $g m_{A}(f, g)$ by

$$
m_{A}(f, g)(s)=c_{f(s) A g(s)}, \quad \text { for all } s \in S .
$$

Recall that two acts $f, g \in \mathscr{F}$ are comotonic if $f(s) \succ f\left(s^{\prime}\right)$ implies $g(s) \succsim g\left(s^{\prime}\right)$ for all $s, s^{\prime} \in S$.

GM 5 (Binary Comonotonic Act Independence) For every essential $A \in \Sigma$, every $B \in \Sigma$, and for all $f, g, h \in \mathscr{F}$ such that $f=x A y, g=x^{\prime} A y^{\prime}$ and $h=x^{\prime \prime} A y^{\prime \prime}$, if $f, g, h$ are pairwise comonotonic, and $x, x^{\prime} \succsim x^{\prime \prime}$ and $y, y^{\prime} \succsim y^{\prime \prime}$ (or $x, x^{\prime} \precsim x^{\prime \prime}$ and $\left.y, y^{\prime} \precsim y^{\prime \prime}\right)$, then

$$
f \succsim g \Longleftrightarrow m_{B}(f, h) \succsim m_{B}(g, h)
$$

The following theorem then holds, where a representation $V$ is said to be subcontinuous if, whenever $\left(f_{\alpha}\right)_{\alpha \in D}$ is a net and $f \in \mathscr{F}$ is such that $f_{\alpha}$ and $f$ are all measurable with respect to the same finite partition, and $f_{\alpha}(s)$ converges to $f(s)$ for all $s \in S$, then $V\left(f_{\alpha}\right) \rightarrow V(f)$.

Theorem 3 (Ghirardato and Marinacci 2001) The following statements are equivalent:

(i) ¿ satisfies axioms GM 1 through GM 5.

(ii) There exist a subcontinuous nontrivial monotonic representation $V: \mathscr{F} \rightarrow \mathbb{R}$ of $\succsim$ and a capacity $\rho: \Sigma \rightarrow[0,1]$ such that $\rho(E) \in(0,1)$ and for all $f \in \mathscr{F}$, all $x \succsim y$ in $X$, all $A \in \Sigma$, letting $u(x):=V(x)$ for all $x \in X$,

$$
V(x A y)=\rho(A) u(x)+(1-\rho(A)) u(y) .
$$


Moreover, the representation $V$ is unique up to positive affine transformations and the capacity $\rho$ is unique.

We now introduce the framework needed to deal with framing effects. Assume that there is a set $\mathscr{A}$ of actions among which the decision maker has to choose, but that he/she can process only if they are framed as acts. This makes sense if we think that in order to make a decision one has to envision different scenarios and the consequences that may follow from choosing a given action in this or that scenario. Now there might be different ways of describing the different relevant scenarios or consequences, so we assume that there are many ways of framing an action as an act. We shall therefore call frame a map $\varphi: \mathscr{A} \rightarrow \mathscr{F}$ and we will consider that the set of available descriptions is a set $\Phi$ of such functions.

Since we hypothesize that the choice of frames carries information relevant to the decision at hand, and granted that in the context of uncertainty information boils down to knowing whether an event will occur or not, we will model decision making conditional on the knowledge of a given event $K$. We shall consider that, conditional on $K$, the decision maker makes his/her decision on the basis of states that belong to $K$, and ignores states that are outside of $K$. Let $\Sigma_{K}$ be the $\sigma$-algebra induced by $\Sigma$ on $K$ (i.e., $B \in \Sigma_{K}$ if $B=A \cap K$ for some $A \in \Sigma$ ) and let $\mathscr{F}_{K}$ be the set of acts defined on $K$ (i.e., simple measurable functions from $\left(K, \Sigma_{K}\right)$ to $\left.X\right)$. We shall consider a binary relation $\succsim$ on $\mathscr{F}$ and shall denote by $\Sigma^{*}$ the set of events that are nonnull with respect to $\succsim$. For each $K \in \Sigma^{*}$, we shall consider a binary relation $\succsim_{K}$ representing the decision maker's preferences on $\mathscr{F}_{K}$, and therefore modeling his/her decision when knowing that $K$ is the case. To simplify notations, for all $f, g \in \mathscr{F}$, when no confusion may arise, we will write $f \succsim_{K} g$ for $f_{\mid K} \succsim_{K} g_{\mid K}, f_{\mid K}$ being the restriction of $f$ to $K$.

We assume the following:

Axiom 1 (Biseparable Preferences-BP)

(i) $\succsim$ satisfies axioms GM 1-GM 5.

(ii) For all $K \in \Sigma^{*}, \succsim_{K}$ satisfies axioms GM 1 and GM 4.

Axiom 2 (Outcome Information Independence-OII) For all $x, y \in X$, for all $K \in$ $\Sigma^{*}, x \succsim_{K} y$ if and only if $x \succsim y$.

This axiom essentially says that for constant acts information does not matter.

We will now define, in the spirit of the previous section, the notion of good news. The definition process will require several steps for the sake of clarity. Given $f \in \mathscr{F}$, we first define the concept of good news for $f$. Given $K \in \Sigma$, write $K^{c}$ the complement of $K$.

Definition 2 Let $f \in \mathscr{F}$ and $K \in \Sigma^{*} . K$ is good news for $f$ if $K^{c} \in \Sigma^{*}$ and there exists $(x, y) \in X^{2}$, call the support of $K$, such that:

(i) $f \sim_{K} x$,

(ii) $f \sim_{K^{c}} y$,

(iii) $x \succ f \succ y$,

We denote $\mathscr{G}(f)$ the set of good news for $f$. 
In other words, $K$ is good news for $f$ if the decision maker is better off learning $K$ while having chosen $f$ than learning $K^{c}$ while having chosen $f$. Notice in particular that, because of condition (iii), $\mathscr{G}(x)=\varnothing$ for $x \in X$, so this basically means that there is nothing to be learned about constant acts.

Now, given $a \in \mathscr{A}, a$ can be expressed by different acts. An event $K$ will be said to be good news for $a$ if it is informative, with the same support, for all the acts by which $a$ can be expressed.

Definition 3 Let $a \in \mathscr{A}$ and $K \in \Sigma^{*} . K$ is good news for $a$ if,

(i) $K \in \mathscr{G}(\varphi(a))$, for all $\varphi \in \Phi$,

(ii) $\varphi(a) \sim_{K} \psi(a)$, for all $\varphi, \psi \in \Phi$,

(iii) $\varphi(a) \sim_{K^{c}} \psi(a)$, for all $\varphi, \psi \in \Phi$.

We denote $\mathscr{K}(a)$ the set of good news for $a$.

Although a theorem in the spirit of the previous section could be proved with the elements already provided, one may wish to impose some structure on the result to be obtained. We consider therefore the following two additional axioms.

Axiom 3 (Non-Atomicity-NA) For all $x, y, z \in X, x \succ z \succ y$ implies that there exists $A \in \Sigma$ such that $z \sim x A y$.

This axiom is the counterpart in our setting of the condition that the Boolean algebra in Bolker-Jeffrey's setting be atomfree.

Axiom 4 (Monotonicity-MON) If for all $f \in \mathscr{F}$, for all $K, K^{\prime} \in \mathscr{G}(f)$, with support $(x, y)$ and $\left(x^{\prime}, y^{\prime}\right)$, respectively, such that $K \subseteq K^{\prime}$, then for all $A \in \Sigma$,

$$
x^{\prime} A y^{\prime} \succsim f \Longrightarrow x A y \succsim f
$$

According to the definition of a good news event, knowing that this event is or is not the case allows to identify the act to one of the outcomes of the support of the event, namely, the good one $x$ or the bad one $y$. The information that the good news is true is all the more valuable because of the larger utility difference between $x$ and $y$. This axiom thus amounts to imposing that knowing smaller good news events is more valuable than knowing larger ones.

Theorem 4 BP and OII hold if and only if there exist a function $V: \mathscr{F} \rightarrow \mathbb{R}$, for each $K \in \Sigma^{*}$, a function $V_{K}: \mathscr{F}_{K} \rightarrow \mathbb{R}$ and for each $f \in \mathscr{F}$ a set function $v(\cdot \mid f): \mathscr{G}(f) \rightarrow[0,1]$ such that,

(i) $V$ is biseparable with willingness to pay $\rho$ and represents $\succsim$;

(ii) $V_{K}$ represents $\succsim_{K}$, with $V_{K}(x)=V(x)$ for all $x \in X$;

(iii) for all $f \in \mathscr{F}$ and $K \in \mathscr{G}(f)$,

$$
V(f)=V_{K}\left(f_{\mid K}\right) v(K \mid f)+V_{K^{c}}\left(f_{\mid K^{c}}\right)(1-v(K \mid f)) .
$$

Moreover, axiom NA and MON hold if and only if 
(i) For all $\alpha \in[0,1]$, there exists $A \in \Sigma$ such that $\rho(A)=\alpha$;

(ii) $v(\cdot \mid f)$ is monotonic.

This theorem shows that invariant biseparable preferences can be written in a form that closely resembles the expected utility-type formula that is characteristic of the Bolker-Jeffrey theorem. It should be noted, however, that, while in the Bolker-Jeffrey framework this formula is always true, for biseparable preferences it is true only on the condition of introducing conditional preferences and only with a certain kind of events. The reason for this fact is that the language of the Bolker-Jeffrey framework is poorer than the standard Savage framework in terms of the number of acts it may contain (it can be argued, indeed, that the Bolker-Jeffrey framework reduces to a Savage framework with only two outcomes).

Now, having retrieved this expected utility form à la Bolker-Jeffrey, it is easy to prove as a corollary, a version in a context of ambiguity of Theorem 2 , that shows that framing effects essentially occur because different frames suggest different likelihoods for good news.

Corollary 1 If axioms BP and OII hold, then for all $a \in \mathscr{A}$ and all $\varphi \in \Phi$, for all $\varphi, \psi \in \Phi$, for all $K \in \mathscr{K}(a)$,

$$
\varphi(a) \succsim \psi(a) \Longleftrightarrow v_{\varphi}(K \mid a) \geq v_{\psi}(K \mid a)
$$

where $v_{\varphi}(\cdot \mid a): \mathscr{K}(a) \rightarrow[0,1]$ is defined by

$$
v_{\varphi}(K \mid a)=v(K \mid \varphi(a))
$$

\section{Moreover, $v_{\varphi}(\cdot \mid a)$ is monotonic if NA and MON hold.}

This corollary shows that Sher and McKenzie's information-based explanation of framing effects does not rely on any kind of implicit Bayesianism (as indeed suggested by their experiments that do not rely on probabilities). All it requires, at least according to this result, is that beliefs be separable from preferences in a well-defined manner.

\section{Concluding Remarks}

Framing effects, i.e., the fact that coreportive sentences are not treated identically in decision-theoretic contexts, ${ }^{8}$ are usually understood as demonstrating that subjects violate one of the most basic axioms of decision theory, namely, extensionality. What this axiom really is, how it is to be understood, in what sense framing effects violate it, and to what extent this violation is to be viewed as irrational are questions that have not, in our opinion, been thoroughly addressed in the literature. In this article, we have attempted to contribute to this discussion in the framework of the BolkerJeffrey decision model and of the Biseparable Preferences model of Ghirardato and Marinacci.

\footnotetext{
8 Irrespective of the fact that they are attributed the same utility (see Le Menestrel and Lemaire 2006).
} 
As we have shown, in the standard Bolker-Jeffrey decision model, extensionality follows basically from an implicit structural assumption embedded in the interpretation of the objects on which preferences are expressed in this model as propositions.

However, we think that framing effects, i.e., violations of extensionality in decision contexts, are normatively grounded. The main reason is that contrary to what is usually asserted, it can be justified to set different utility values on coreportive sentences, to the extent that these sentences provide different clues as to the likelihood of events confirming that we have made the right choice, as McKenzie and Nelson (2003)'s experiments have made clear.

In order, therefore, to allow for framing effects in the Bolker-Jeffrey model, we introduce some slight modification of the structure of the underlying set and of its interpretation, making room for a precise definition of coreportiveness. This modification leads to a formalization of the intuition discussed above, therefore showing that this intuition follows directly from a rigorous analysis of the modified Bolker-Jeffrey decision model that we propose.

While the Bolker-Jeffrey model is essentially an instance of the Bayesian decision theory, there is no reason to believe that the analysis of framing effects done in this context strongly relies on core Bayesian assumptions. Indeed, we show that, in a standard Savagean framework, it is possible, at the cost of introducing a family of conditional preference relations, to extend the results presented here to the case of ambiguity, where beliefs are represented by a nonadditive probability or capacity.

This research leads to broader questions.

The first relates to the nature and status of anomalies in decision theory. It has been taken for granted in the last two decades that, contrary to, say, violations of expected utility like the Ellsberg paradox, these anomalies could not be incorporated into a rational account of decision making, and were evidence to the fact that a normatively sound and descriptively accurate decision theory was impossible. It seems to us that the results presented here cast some doubt on this claim, and give some hope that these anomalies may be treated as rigorously and successfully as the Ellsberg paradox and ambiguity aversion have been in the last decades. At a deeper level, though, the question of the proper norm that should be used to classify anomalies seems to us an open and interesting question.

Second, we would like to deepen our understanding of the way one could elude extensionality in the Bolker-Jeffrey decision model, doing away with this implicit axiom altogether. It seems to us that this would entail working with structures more general than Boolean algebras, which from a technical point of view is far from trivial. Moreover, in our representation theorem, there is no primitive definition of informational equivalence: informational equivalence is a revealed notion. It would be interesting to explore, from a syntactical point of view, this notion in propositional logic.

Acknowledgments The authors gratefully acknowledge the deep comments of John Broome which greatly contributed to the improvement of this article. We also thank Ethan Bolker, Giacomo Bonanno, Luc Bovens, and Mei Wang. 


\section{Appendix A: Proof of Theorem 2}

The proof proceeds in several steps.

Step 1. By Bolker's theorem, there exists a function $U: \mathbb{P} \rightarrow \mathbb{R}$ and a probability measure $P: \mathbb{P} \rightarrow[0,1]$ such that

(a) $U(p) \geq U\left(p^{\prime}\right) \Leftrightarrow p \succsim p^{\prime}$.

(b) For all $p, p^{\prime} \in \mathbb{P}, U(p)=U\left(p \wedge p^{\prime}\right) P\left(p^{\prime} \mid p\right)+U\left(p \wedge \neg p^{\prime}\right) P\left(\neg p^{\prime} \mid p\right)$, where

$$
P\left(p^{\prime} \mid p\right):=\frac{P\left(p^{\prime} \wedge p\right)}{P(p)} .
$$

Step 2. Let $\varphi \in \Phi, e \in \mathscr{E}$. Define:

$$
P_{\varphi}(e):=P(\varphi(e))
$$

Let us show that $P_{\varphi}$ is a finitely additive probability measure.

First, because $\varphi$ is a morphism, $\varphi(\top)=\top$, therefore, $P_{\varphi}(\top)=P(\top)=1$.

Second, for disjoint $e$ and $e^{\prime}$,

$$
\begin{aligned}
P_{\varphi}\left(e \vee e^{\prime}\right) & =P\left(\varphi\left(e \vee e^{\prime}\right)\right)=P\left(\varphi(e) \vee \varphi\left(e^{\prime}\right)\right)=P(\varphi(e))+P\left(\varphi\left(e^{\prime}\right)\right) \\
& =P_{\varphi}(e)+P_{\varphi}\left(e^{\prime}\right) .
\end{aligned}
$$

Step 3. For all $e \in \mathscr{E}, k \in \mathscr{K}(e), \varphi, \varphi^{\prime} \in \Phi$, we have, using the fact that $\varphi$ and $\varphi^{\prime}$ are morphisms:

$$
\begin{aligned}
\varphi(e) & \succsim \varphi^{\prime}(e) \Longleftrightarrow U(\varphi(e)) \geq U\left(\varphi^{\prime}(e)\right) \\
& \Longleftrightarrow U(\varphi(e) \wedge \varphi(k)) P(\varphi(k) \mid \varphi(e))+U(\varphi(e) \wedge \neg \varphi(k)) P(\neg \varphi(k) \mid \varphi(e)) \\
& \geq U\left(\varphi^{\prime}(e) \wedge \varphi^{\prime}(k)\right) P\left(\varphi^{\prime}(k) \mid \varphi^{\prime}(e)\right) \\
& +U\left(\varphi^{\prime}(e) \wedge \neg \varphi^{\prime}(k)\right) P\left(\neg \varphi^{\prime}(k) \mid \varphi^{\prime}(e)\right) \\
& \Leftrightarrow U(\varphi(e \wedge k)) P_{\varphi}(k \mid e)+U(\varphi(e \wedge \neg k)) P_{\varphi}(\neg k \mid e) \\
& \geq U\left(\varphi^{\prime}(e \wedge k)\right) P_{\varphi^{\prime}}(k \mid e)+U\left(\varphi^{\prime}(e \wedge \neg k)\right) P_{\varphi^{\prime}}(\neg k \mid e) \\
\Leftrightarrow & P_{\varphi}(k \mid e) \geq \frac{U\left(\varphi^{\prime}(e \wedge k)\right)-U\left(\varphi^{\prime}(e \wedge \neg k)\right)}{U(\varphi(e \wedge k))-U(\varphi(e \wedge \neg k))} P_{\varphi^{\prime}}(k \mid e) \\
& +\frac{U\left(\varphi^{\prime}(e \wedge \neg k)\right)-U(\varphi(e \wedge \neg k))}{U(\varphi(e \wedge k))-U(\varphi(e \wedge \neg k))} \\
\Leftrightarrow & P_{\varphi}(k \mid e) \geq P_{\varphi^{\prime}}(k \mid e),
\end{aligned}
$$

where the last two lines follow from the fact that $k$ belongs to $\mathscr{K}(e)$. 


\section{Appendix B: Proof of Theorem 4 and Corollary 1}

\section{Proof (Theorem 4)}

Step 1. By axiom BP and Theorem 3, there exist a monotonic and subcontinuous $V$ and a capacity $\rho$ satisfying conditions 1 and 2 . Moreover, the second part of axiom $B P$ implies that for any $f \in \mathscr{F}$ and $K \in \Sigma^{*}$, there exists $c_{f}^{K} \in X$ such that $f_{\mid K} \sim_{K} c_{f}^{K}$ (Ghirardato and Marinacci 2001, Lemma 29). By axiom OII, we can define $V_{K}(f)=u\left(c_{f}^{K}\right)$. Then $V_{K}$ represents $\succsim_{K}$ and satisfies $V_{K}(x)=V(x)$ for any $x \in X$.

Step 2. Let $f \in \mathscr{F}$ and $K \in \mathscr{G}(f)$. Then there exist $x, y \in X$ such that:

(i) $f \sim_{K} x$,

(ii) $f \sim_{K^{c}} y$,

(iii) $x \succ f \succ y$,

Therefore, $u(x)>V(f)>u(y)$ and there exists a unique $\alpha(K, f) \in(0,1)$ such that $V(f)=\alpha(K, f) u(x)+(1-\alpha(K, f)) u(y)$. Define $v(K \mid f):=$ $\alpha(K, f)$. Moreover, $u(x)=V_{K}\left(f_{\mid K}\right)$ and $u(y)=V_{K^{c}}\left(f_{\mid K^{c}}\right)$ by construction of $V_{K}$ and $V_{K^{c}}$.

Step 3. Let us deal now with the second part of the theorem.

First, let $\alpha \in(0,1)$. Let $f, g$ be such that $f \succ g$. They exist by axiom BP. Normalize $u$ so that $u\left(c_{f}\right)=1$ and $u\left(c_{g}\right)=0$. Then $u\left(c_{f}\right)>\alpha>u\left(c_{g}\right)$. Since $u$ is continuous and $X$ is connected, there exists $z \in X$ such that $u(z)=$ $\alpha$. Therefore, by axiom NA, there exists $A \in \Sigma$ such that $\alpha=u(z)=$ $V\left(c_{f} A c_{g}\right)=\rho(A)$.

Conversely, let $x, y, z$ be such that $x \succ z \succ y$. Then, there exists $\alpha \in(0,1)$ be such that $u(z)=\alpha u(x)+(1-\alpha) u(y)$. Now there exists $A \in \Sigma$ such that $\rho(A)=\alpha$, hence $u(z)=V(x A y)$.

Now, let $K, K^{\prime} \in \mathscr{G}(f)$ be such that $K \subseteq K^{\prime}$. By axiom NA, there exists $A^{\prime}$ such that $c_{f} \sim x^{\prime} A^{\prime} y^{\prime},\left(x^{\prime}, y^{\prime}\right)$ being the support of $K^{\prime}$. Then, since $\succsim$ is biseparable, $v\left(K^{\prime} \mid f\right)=\rho\left(A^{\prime}\right)$. Similarly, there exists $A$ such that $c_{f} \sim x A y$, $(x, y)$ being the support of $K$ and $v(K \mid f)=\rho(A)$. Now, by axiom MON, $x A^{\prime} y \succsim f \sim x^{\prime} A^{\prime} y^{\prime}$, and, since $x^{\prime} A^{\prime} y^{\prime} \sim f \sim x A y$, this implies that $x A^{\prime} y \succsim x A y$, and therefore $\rho\left(A^{\prime}\right) \geq \rho(A)$, i.e., $v(K \mid f) \leq v\left(K^{\prime} \mid f\right)$.

Conversely, assume that $v(K \mid f) \leq v\left(K^{\prime} \mid f\right)$ whenever $K, K^{\prime} \in \mathscr{G}(f)$ and $K \subseteq K^{\prime}$. Then, let $A$ be such that $x^{\prime} A y^{\prime} \succsim f$. Then $u\left(x^{\prime}\right) \rho(A)+u\left(y^{\prime}\right)$ $(1-\rho(A)) \geq V(f)$, hence

$$
\rho(A) \geq \frac{V(f)-u\left(y^{\prime}\right)}{u\left(x^{\prime}\right)-u\left(y^{\prime}\right)}=v\left(K^{\prime} \mid f\right) \geq v(K \mid f)=\frac{V(f)-u(y)}{u(x)-u(y)},
$$

therefore $u(x) \rho(A)+u(y)(1-\rho(A)) \geq V(f)$, i.e., $x A y \succsim f$. 


\section{Proof (Corollary 1)}

It follows from Theorem 4 and from the definition of good news for $a$ that for all $a \in \mathscr{A}$, for all $K \in \mathscr{K}(a)$, for all $\varphi, \psi \in \Phi$,

$$
\begin{aligned}
\varphi(a) & \succsim \psi(a) \\
& \Leftrightarrow\left(V_{K}\left(\varphi(a)_{\mid K}\right)-V_{K^{c}}\left(\varphi(a)_{\mid K^{c}}\right)\right) v(K \mid \varphi(a))+V_{K^{c}}\left(\varphi(a)_{\mid K^{c}}\right) \\
& \geq\left(V_{K}\left(\psi(a)_{\mid K}\right)-V_{K^{c}}\left(\psi(a)_{\mid K^{c}}\right)\right) v(K \mid \psi(a))+V_{K^{c}}\left(\psi(a)_{\mid K^{c}}\right) \\
& \Leftrightarrow\left(V_{K}\left(\varphi(a)_{\mid K}\right)-V_{K^{c}}\left(\varphi(a)_{\mid K^{c}}\right)\right) v(K \mid \varphi(a))+V_{K^{c}}\left(\varphi(a)_{\mid K^{c}}\right) \\
& \geq\left(V_{K}\left(\varphi(a)_{\mid K}\right)-V_{K^{c}}\left(\varphi(a)_{\mid K^{c}}\right)\right) v(K \mid \psi(a))+V_{K^{c}}\left(\varphi(a)_{\mid K^{c}}\right) \\
& \Leftrightarrow v(K \mid \varphi(a)) \geq v(K \mid \psi(a)) .
\end{aligned}
$$

\section{References}

Ahn, D. S., \& Ergin, H. (2007). Framing contingencies. Unpublished Manuscript, University of California, Berkeley.

Arrow, K. J. (1982). Risk perception in psychology and economics. Economic Enquiry, 20, 1-9.

Cohen, M., \& Jaffray, J.-Y. (1980). A theory of decision under complete ignorance. Econometrica, 48(5), 1281-1300.

Ghirardato, P., \& Marinacci, M. (2001). Risk, ambiguity, and the separation of utility and beliefs. Mathematics of Operations Research, 26, 864-890.

Gilboa, I. (Ed.). (2004). Uncertainty in economic theory: Essays in Honor of David Schmeidler's 65th Birthday. London: Routledge.

Giraud, R. (2004). Framing under risk: Endogenizing the reference point and separating cognition and decision. Cahiers de la MSE Bla04090. Université Paris I, France.

Gold, N., \& List, C. (2004). Framing as path-dependence. Economics and Philosophy, 20(2), 253-277.

Kahneman, D., \& Tversky, A. (1979). Prospect theory: An analysis of decision under risk. Econometrica, 47, 263-291.

Koppelberg, S. (1989). General theory of Boolean algebras. In J. D. Monk \& R. Bonnet (Eds.), Handbook of Boolean algebras. Amsterdam: North Holland.

Kühberger, A. (1998). The influence of framing on risky decisions: A meta-analysis. Organizational Behavior and Human Decision Processes, 75(1), 23-55.

Le Menestrel, M., \& Lemaire, B. (2006). Ratio-scale measurement with intransitivity and incompleteness: The homogeneous case. Theory and Decision, 60, 207-217.

Le Menestrel, M., \& Van Wassenhove, L. (2001). The domain and interpretation of utility functions: An exploration. Theory and Decision, 51, 329-349.

Levin, I. P., Schneider, S. L., \& Gaeth, G. J. (1998). All frames are not created equal: A typology and critical analysis of framing effects. Organizational Behavior and Human Decision Processes, 76(1), 149-188.

Lipman, B. (1999). Decision theory without logical omniscience: Towards an axiomatic framework for bounded rationality. Review of Economic Studies, 66, 339-361.

Malinvaud, E. (1952). Note on the von Neumann-Morgenstern's strong independence axiom. Econometrica, $60,679$.

McKenzie, C. R. M., \& Nelson, J. D. (2003). What a speaker's choice of frame reveals: Reference points, frame selection, and framing effects. Psychonomic Bulletin and Review, 10(3), 596-602.

Quattrone, G. A., \& Tversky, A. (2000). Constrasting rational and psychological analyses of political choice. In: D. Kahneman \& A. Tversky (Eds.), Choice, values and frames. Cambridge: Cambridge University Press.

Rubinstein, A. (2000). Modeling bounded rationality. Cambridge: MIT Press. 
Sen, A. (1986). Information and invariance in normative choice. In W. P. Heller, R. M. Starr, \& D. A. Starret (Eds.), Social choice and public decision making (Essays in Honor of Kenneth J. Arrow, Vol. 1). Cambridge: Cambridge University Press.

Sher, S., \& McKenzie, C. R. M. (2006). Information leakage from logically equivalent frames. Cognition, 101, 467-494.

Tversky, A., \& Kahneman, D. (1992). Advances in prospect theory: Cumulative representation of uncertainty. Journal of Risk and Uncertainty, 5, 297-323.

Tversky, A., \& Kahneman, D. (2000). Rational choice and the framing of decisions. In D. Kahneman \& A. Tversky (Eds.), Choice, values and frames. Cambridge: Cambridge University Press.

Tversky, A., Simonson, I., \& Shafir, E. (2000). Reason-based choice. In D. Kahneman \& A. Tversky (Eds.), Choice, values and frames. Cambridge: Cambridge University Press. 